\title{
ON POTENT RINGS
}

\author{
BINGJUN LI
}

\begin{abstract}
A ring $R$ is called an $I_{0}$-ring if each left ideal not contained in the Jacobson radical $J(R)$ contains a non-zero idempotent. If, in addition, idempotents can be lifted modulo $J(R), R$ is called an $I$ ring or a potent ring. We study whether these properties are inherited by some related rings. Also, we investigate the structure of potent rings.
\end{abstract}

\section{Introduction}

Let $R$ be an associative ring possibly without an identity. Following Nicholson [7], $R$ is said to be an $I_{0}$-ring if each left ideal $L$ of $R$ not contained in the Jacobson radical $J(R)$ contains a nonzero idempotent. It was proved that this definition is left-right symmetric [7, Lemma 1]. If, in addition, idempotents can be lifted modulo $J(R), R$ is called an $I$-ring or a potent ring.

Warfield [10] introduced the class of exchange rings. He called a ring $R$ an exchange ring if $R_{R}$ has the exchange property introduced by Crawley and Jonsson [3], and Nicholson proved that every exchange ring is potent [8, Proposition 1.9]. So the class of potent rings is quite large. Call a ring $R$ semi-regular (semi$\pi$-regular, semi-strongly $\pi$-regular) if $R / J(R)$ is regular $(\pi$-regular, strongly $\pi$-regular) and idempotents can be lifted modulo $J(R)$. It is known in the literature that local rings, semiperfect rings, semi-regular rings, semi-strongly $\pi$-regular rings and semi- $\pi$-regular rings (in the order of containment) are all potent rings, while there still exist potent rings which belong to none of the above classes [8, Example 1.7].

Nicholson [7, Proposition 1.6] proved that if $R$ is potent so is each one-sided ideal of $R$. He [7, Proposition 1.8] proved that if $R$ is an $I_{0}$-ring so is the ring $M_{n}(R)$ of all $n \times n$ matrix over $R$. Also he proved that primitive idempotents in an $I_{0}$-ring can always be lifted modulo $J(R)$ [7, Lemma 2.5].

In section 2 , we study whether the properties of potent rings are inherited by some related rings. We prove that $R / \sqrt{0}$ is an $I_{0}$-ring if and only if $R$ is an $I_{0}$-ring (where $\sqrt{0}$ is nil radical of $R$ ); $R$ is potent if and only if formal power

Received October 6, 2007.

2000 Mathematics Subject Classification. Primary 16D10, 16D60.

Key words and phrases. potent rings, idempotents, Jacobson radical, formal power series ring.

This work was supported partially by NCET.

(C) 2008 The Korean Mathematical Society 
series ring $R[[x]]$ is potent. However, we show that the polynomial ring $R[x]$ is never potent for any ring $R$ with $J(R)=0$.

The general structure of potent rings is still undetermined. A problem one might have in such an attempt is that the usual chain conditions on rings (e.g. Artinian, Noetherian) are too strong for potent rings. In fact, by a theorem of Nicholson [7, Theorem 4.3], potent rings containing no infinite set of orthogonal idempotents are just semiperfect rings. In section 3 , we investigate the structure of potent rings, and prove that a potent ring which has only trivial idempotents is precisely a local ring.

Throughout this paper all rings are assumed to be associative with identity and modules are unitary. $J(R), U(R)$ always stand for, respectively, the Jacobson radical, the group of units of a ring $R$. $\sqrt{0}$ denotes the nil radical of $R, M_{n}(R)$ denotes the $n \times n$ matrix ring over the ring $R$. For a left ideal $I$ of $R, \pi: R \rightarrow R / I$ will denote the natural quotient ring homomorphism, and we will write $\pi(r)=\bar{r}$ for any $r \in R$.

\section{Some properties of potent rings}

It turns out that many of the basic properties of exchange rings hold for potent rings. We first list here in Proposition 2.1 several properties of potent rings. All of them can be found in [7], so the proofs are omitted here.

Proposition 2.1. (1) $R$ is a potent ring if and only if $R / J(R)$ is a potent ring and idempotents can be lifted modulo $J(R)$.

(2) If $R$ is potent so is each one-sided ideal of $R$.

(3) If $R$ is potent so is each subring $a R b$ where $a, b \in R$.

(4) $A$ direct sum of rings is potent if and only if the same is true for each summand.

Corollary 2.2. (1) Let $R$ be a potent ring and $e^{2}=e \in R$, then the ring eRe is also potent.

(2) $R$ is an $I_{0}$-ring if and only if $M_{n}(R)$ is an $I_{0}$-ring.

Proof. (1) Let $a=b=e=e^{2}$, the result follows from Proposition 2.1(3).

(2) Assume $M_{n}(R)$ is an $I_{0}$-ring. Let $E_{i j}(1 \leq i, j \leq \mathrm{n})$ be the matrix units. Obviously, $E_{11}$ is an idempotent in $M_{n}(R)$. Now the result follows from (1) since $E_{11} M_{n}(R) E_{11} \simeq R$ as a ring.

Conversely, let $L$ be a left ideal of $M_{n}(R)$ with $L$ not contained in $J\left[M_{n}(R)\right]$ $=M_{n}[J(R)]$. There exists a matrix $A=\left(a_{i j}\right) \in L$ with $a_{p q}$ not in $J(R)$ for some $p, q$. Then $L_{0}=\left\{x \in R \mid x=x_{p q}\right.$ for some $\left.\left(x_{i j}\right) \in L\right\}$ is a left ideal of $R$ which is not contained in $J(R)$. Pick $0 \neq e^{2}=e \in L_{0}$, and let $X=\left(x_{i j}\right) \in L$ with $x_{p q}=e$. It is straightforward to verify that $E_{p q}(e) X=\sum_{j} E_{q j}\left(e x_{p j}\right)$ is a nonzero idempotent in $L$.

Lemma 2.3. For a ring $R$, let $L$ be a left ideal of $R, T$ be a ideal of $R$ with $T \subseteq J(R)$ and let $x \in L$. If there is an idempotent $f$ such that $f-x \in T$, then there exists an idempotent $e \in L$ such that $e-x \in T$. 
Proof. If $f^{2}=f$ and $f-x \in T \subseteq J(R)$, let $a=(f-x)(f-x-1)^{-1}$, then $a+(f-x)=a(f-x)$. Hence $f a f+f-f x f=f a f-f a x f$ so $f=f(x-a x) f$. Take $e=f(x-a x)$, as desired.

Proposition 2.4. $R$ is an $I_{0}$-ring if and only if so is $R / \sqrt{0}$.

Proof. Any left ideal of $R / \sqrt{0}$ has the form $L / \sqrt{0}$ where $L$ is a left ideal of $R$ containing $\sqrt{0}$. If $0 \neq e=e^{2} \in L$, then $0 \neq \bar{e}=\bar{e}^{2} \in R / \sqrt{0}$ since the only idempotent $\alpha \in \sqrt{0}$ is $\alpha=0$. Conversely, Let $L$ be a left ideal of $R$ with $L \nsubseteq J(R)$, then $(L+\sqrt{0}) / \sqrt{0}$ is a nonzero left ideal of $R / \sqrt{0}$ since $\sqrt{0} \subseteq J(R)$. We have $0 \neq \bar{t}=\bar{t}^{2}=\overline{x+y} \in(L+\sqrt{0}) / \sqrt{0}$ for some $x \in L, y \in \sqrt{0}$. There exists $f=f^{2} \in R$ such that $\bar{f}=\bar{t}$ since idempotents can always be lifted modulo $\sqrt{0}$, so we get $f-x \in \sqrt{0}$. Then there exists an idempotent $e \in L$ such that $e-x \in \sqrt{0}$ by Lemma 2.3. If $e=0$, then $x \in \sqrt{0}$, hence $\bar{t}=0$, a contradiction. We conclude that $L$ contains a nonzero idempotent.

We now explore more properties of potent rings. If $R$ and $S$ are potent rings, it is natural to expect that the formal triangular matrix ring and the $n \times n$ upper triangular matrix ring over $R$ and also polynomial ring are potent rings.

Proposition 2.5. Let $R, S$ be two rings, and $M$ be an $(R, S)$-bimodule. Let

$$
E=\left(\begin{array}{cc}
R & M \\
0 & S
\end{array}\right)
$$

be the formal triangular matrix ring. Then $E$ is potent if and only if $R$ and $S$ are potent.

Proof. Let $e_{i i}$ be matrix units for $i=1,2$. Then $e_{11} E e_{11} \simeq R$ and $e_{22} E e_{22} \simeq S$, hence the result follows from Proposition 2.1(4) and Corollary 2.2(1).

Denote the upper triangular matrix ring of $R$ by $T_{n}(R)$. The following proposition is an analogous result of C. Y. Hong et [5, Proposition 2.2]. The proof to it is also similar, so we omit the proof here.

Proposition 2.6. The following statements are equivalent:

(1) $R$ is a potent ring.

(2) $T_{n}(R)$ is a potent ring.

It is well known that the polynomial rings over nonzero exchange rings are never exchange rings.

Question: If $R$ is a potent ring, does it follow that the polynominal ring $R[x]$ is a potent ring?

Unfortunately, this has a negative answer in general as the following statement shows:

For any $\operatorname{ring} R$, let $T=\left\{t_{i}: i \in I\right\}$. The following result of Amitsur [6, Theorem 5.10] describes the structure of the radical of $S=R[T]$. 
Amistsur's Theorem. Let $R$ be any ring, $T=\left\{t_{i}: i \in I\right\}$ be a nonempty set of commuting independent variables, and $S=R[T]$. Let $J=\operatorname{rad} S$ and $N=R \bigcap J$. Then $N$ is a nil ideal in $R$, and $J=N[T]$.

Note that if a left (resp., right) ideal $U \subseteq R$ is nil, then $U \subseteq J(R)$. The theorem above has the following pleasant consequence.

Corollary 2.7. If $R$ is semiprimitive (i.e., $J(R)=0$ ), then $R[x]$ is also semiprimitive.

Suppose that $R$ is a nonzero potent ring with $J(R)=0$, then right ideal $x R[x]$ is not contained in $J(R[x])$. But $x R[x]$ contains the only idempotent 0 , hence $R[x]$ is not a potent ring.

Using the similar argument of [5, Proposition 2.3], we have the following.

Proposition 2.8. Let $R$ be a potent ring, then $R[x] /\left\langle x^{n+1}\right\rangle$ is potent for any $n \geq 1$. The converse holds if idempotents in $R / J(R)$ can be lifted to $R$.

Proof. Assume that $R$ is potent, and write $u=\bar{x}=x+\left\langle x^{n+1}\right\rangle$, then

$$
R[x] /\left\langle x^{n+1}\right\rangle=R[u]=R+R u+\cdots+R u^{n+1}
$$

with $u^{n+1}=0$. It is not difficult to prove that $J(R[u])=J(R)+\langle u\rangle$, where $\langle u\rangle$ denotes the ideal of $R[u]$ generated by $u$. Hence $R[u] / J(R[u]) \simeq R / J(R)$ is potent by Proposition 2.1.

Now we claim that idempotents can be lifted modulo $J(R[u])$. Let $f+$ $J(R[u])$ be an idempotent in $R[u]$, then $f=a_{0}+a_{1} u+\cdots+a^{n} u^{n}$ for some $a_{0}, a_{1}, \ldots, a_{n} \in R$. Note that $J(R[u])=J(R)+\langle u\rangle$, therefore, $\left(a_{0}+J(R[u])\right)^{2}=$ $a_{0}^{2}+J(R[u])=a_{0}+J(R[u])$. Hence $a_{0}^{2}-a_{0} \in J(R)$. Since idempotents can be lifted modulo $J(R)$, we get $e-a_{0} \in J(R) \subseteq J(R[u])$ for some $e^{2}=e \in R$, as desired.

Conversely, suppose that $R[x] /\left\langle x^{n+1}\right\rangle$ is a potent ring, then $R / J(R)$ is also potent since $R[u] / J(R[u]) \simeq R / J(R)$. Now the result follows from Proposition 2.1 .

Recall that for a ring $R$ with a ring endomorphism $\alpha: R \rightarrow R$, the skew power series ring $R[[x ; \alpha]]$ of $R$ is the ring obtained by giving the formal power series ring over $R$ with the new multiplication $x r=\alpha(r) x$ for all $r \in R$. We are going to investigate when is $R[[x ; \alpha]]$ potent? The following lemma will be needed.

Lemma 2.9. If $e=a_{0}+a_{1} x+\cdots \quad$ is a nonzero idempotent in $R[[x ; \alpha]]$, then $a_{0} \neq 0$ for any ring endomorphism $\alpha$.

Proof. Assume on the contrary $a_{0}=0$ and let $k=\min \left\{n \mid a_{n} \neq 0\right\} \geq 1$. Then

$$
\begin{aligned}
e^{2} & =\left(a_{k} x^{k}+a_{k+1} x^{k+1}+\cdots\right)^{2} \\
& =a_{k} \alpha^{k}\left(a_{k}\right) x^{2 k}+\left(a_{k+1} \alpha^{k+1}\left(a_{k}\right)+a_{k} \alpha^{k}\left(a_{k+1}\right)\right) x^{2 k+1}+\cdots .
\end{aligned}
$$

Therefore, $e$ can not be equal to $e^{2}$, a contradiction. 
In particular, when $\alpha=1_{R}$, we have the same result in formal power series ring $R[[x]]$.

Proposition 2.10. Let $\alpha$ be an endomorphism of $R$. Then the following statements are equivalent.

(1) $R$ is a potent ring.

(2) The formal power series ring $R[[x]]$ of $R$ is potent.

(3) The skew power series ring $R[[x ; \alpha]]$ of $R$ is potent.

Proof. (1) $\Rightarrow(2)$ : It is easy to check that $f=a_{0}+a_{1} x+\cdots$ is a unit in $R[[x]]$ if and only if $a_{0}$ is a unit in $R$, so $J(R[[x]])=J(R)+R[[x]] x$. Hence $R[[x]] / J(R[[x]]) \simeq R / J(R)$ is potent by Proposition 2.1 .

We claim that idempotents can be lifted modulo $J(R[[x]])$. Let $h+J(R[[x]])$ be an idempotent in $R[[x]]$, say $h=b_{0}+b_{1} x+\cdots$ for some $b_{0}, b_{1}, \ldots \in R$. We have $\left(b_{0}+J(R[[x]])^{2}=b_{0}^{2}+J(R[[x]])=b_{0}+J(R[[x]])\right.$. Hence $b_{0}^{2}-b_{0} \in J(R)$. Since idempotents can be lifted modulo $J(R)$, we have $e-b_{0} \in J(R)$ for some $e^{2}=e \in R$. Let $t=t^{2}=e+0 x+0 x^{2}+\cdots \in R[[x]]$, then $t-f \in J(R[[u]])$. The result follows from proposition 2.1.

$(2) \Rightarrow(1)$ : If $L$ is a left ideal of $R$ not contained in $J(R)$, it is easy to verify that $L+R[[x]] x$ is also a left ideal of $R[[x]]$ not contained in $J(R[[x]])$. There exists a nonzero idempotent $f=f^{2}=r+c_{1} x+c_{2} x^{2}+\cdots \in L+R[[x]] x$ by assumption, and we have $r \neq 0$ by a special case in Lemma 2.9. Then we get $0 \neq r=r^{2} \in L$, as required. Let $a^{2}-a \in J(R)$, then it is easy to see that $a+J(R[[x]])$ is an idempotent in $R[[x]] / J(R[[x]])$. There exists an idempotent $h=h^{2}=w_{0}+w_{1} x+w_{2} x^{2}+\cdots \in R[[x]]$ such that $h-a \in J(R[[x]])=$ $J(R)+R[[x]] x$ since $R[[x]]$ is potent. So $w_{0}-a \in J(R)$ with $w_{0}=w_{0}^{2} \in R$, so idempotents can be lifted modulo $J(R)$. Hence $R$ is potent.

$(2) \Leftrightarrow(3)$ : We have $U(R[[x ; \alpha]])=\left\{a_{0}+a_{1} x+\cdots: a_{0} \in U(R)\right\}$ without any assumption on the endomorphism $\alpha$. Hence the equivalence follows since $J(R[[x]])=J(R[[x ; \alpha]])$

However, the class of potent rings is not closed under homomorphic image [7, Example 1.9].

\section{The structure of potent rings}

Recall that a ring $R$ is called to be abelian if every idempotent in $R$ is central. A ring $R$ is called to be reduced if $R$ has no nonzero nilpotent, or equivalently, if $a^{2}=0 \Rightarrow a=0$ in $R$.

Lemma 3.1. If $R$ is a potent ring with $J(R)=0$, then the following statements are equivalent.

(1) $R$ is reduced.

(2) $R$ is abelian.

Proof. (1) $\Rightarrow(2)$ : Let $e$ be an idempotent in $R$, then $(\text { ere }-r e)^{2}=0=$ $(e r e-e r)^{2}$ for all $r \in R$. Hence $e r=e r e=r e$ since $R$ is reduced. 
$(2) \Rightarrow(1)$ : If $0 \neq x \in R$, then $R x$ is not contained in $J(R)$. By definition, there exists a nonzero idempotent $e=e^{2}=r x$ for some $r \in R$. We have $e=e r x=r e x=r^{2} x^{2}$ since $e$ is central, hence $x^{2} \neq 0$.

A ring $R$ is called to be strongly $\pi$-regular if for any $a \in R$, there exists a positive integer $n$, depending on $a$, such that $a^{n}=a^{n+1} x$. A ring $R$ is called to be strongly regular if for any $a \in R$, there exists some $x \in R$, such that $a^{2} x=a$.

Theorem 3.2. If $R$ is an abelian potent ring. Let $\bar{R}=R / J(R)$, then the following statements are equivalent.

(1) $\bar{R}$ is strongly $\pi$-regular.

(2) $\bar{R}$ is strongly regular.

Proof. $(2) \Rightarrow(1)$ : is a tautology.

$(1) \Rightarrow(2)$ : Assume that $\bar{R}$ is strongly $\pi$-regular, then $x^{n}=x^{n+1} r$ for any $x \in$ $\bar{R}$ and some $n \geq 1$ and some element $r \in \bar{R}$ commuting with $x$ (see Azumaya [1]). Therefore, $\left(x-x^{2} r\right)^{n}=x^{n}(1-x r)^{n}=\left(x^{n}-x^{n+1} r\right)(1-x r)^{n-1}=0$. Since $R$ is potent, so is $\bar{R}$. By the virtue of Lemma 3.1, $\bar{R}$ is reduced. Hence we obtain $x-x^{2} r=0$, as desired.

The following lemma is partly due to Nicholson [7, Proposition 2.1].

Lemma 3.3. The following conditions are equivalent for an idempotent $e$ in an $I_{0}$-ring $R$.

(1) $e$ is primitive.

(2) $e$ is local.

(3) $\bar{e}$ is right irreducible in $\bar{R}=R / J(R)$.

Proof. (1) $\Rightarrow(2)$ : If $a \in e R e$ and $a \bar{\epsilon} J(e R e)=e R e \bigcap J(R)$, then $a \bar{\epsilon} J(R)$. Therefore, there exists a nonzero idempotent $f=f^{2} \in R a \subseteq R e$. Then $R f=R e=R a$ since $e$ is primitive. Hence we can write $e=e r e a$ for some $r \in R$ since $e a=e$. Therefore, $a$ has a left inverse in $e R e$, so $e$ is local.

$(2) \Rightarrow(1)$ : Trivial.

(3) $\Leftrightarrow(2): \bar{e}$ is right irreducible if and only if $\bar{e} R \bar{e}$ is a division ring. Note that $\bar{e} R \bar{e} \simeq e R e / J(e R e)$, then $\bar{e} R \bar{e}$ is a division ring if and only if $e R e$ is a local ring. This gives the equivalence of (2) and (3).

Theorem 3.4. For a potent ring $R$, the following are equivalent:

(1) $R$ contains no infinite set of orthogonal idempotents.

(2) $1=e_{1}+\cdots+e_{n}$ with $e_{i} s$ primitive orthogonal idempotents.

(3) $R$ is semiperfect.

Proof. $(1) \Rightarrow(2)$ : Trivial.

$(2) \Rightarrow(3)$ : By Lemma 3.3, primitive idempotents of a potent ring are local idempotents. Then $R$ is semiperfect by Lam [6, Theorem 23.6].

$(3) \Rightarrow(1)$ : It is well known that semiperfect rings contain no infinite set of orthogonal idempotents. 
At the end of this paper, we will investigate potent rings which have only trivial idempotents.

Theorem 3.5. Let $R$ be a potent ring whose idempotents are trivial, then $R$ is precisely a local ring.

Proof. It is well known that local ring has only trivial idempotents and $R=$ $U(R) \cup J(R)$. Idempotents $\overline{0}, \overline{1}$ in division ring $R / J(R)$ can be lifted to $0,1 \in R$, respectively. For any left ideal $L \nsubseteq J(R)$, then $L$ contains a unit of $R$. Hence $0 \neq 1=1^{2} \in L$, as desired.

Conversely, for any $a \bar{\epsilon} J(R)$, there exists a nonzero idempotent $e \in R a$, hence $a$ is left invertible since $e=1$. If $M \neq N$ are maximal left ideals of $R$, then $M+N=R$. We have $m+n=1$ for some $m \in M, n \in N$. Obviously, $m$ and $n$ can not be contained in $J(R)$ at the same time since $1 \bar{\epsilon} J(R)$. We may assume $m \bar{\epsilon} J(R)$, then $m$ is left invertible, hence $M=R$, a contradiction. We conclude that $R$ has only one maximal left ideal, so $R$ is a local ring.

Acknowledgment. The author is grateful to the referee for his/her careful reading of the paper and for the helpful suggestions which improved this paper.

\section{References}

[1] G. Azumaya, Strongly $\pi$-regular ring, J. Fac. Sci. Hokkaido Univ. 13 (1954), 34-39.

[2] V. P. Camillo and H. P. Yu, Exchange rings, units and idempotents, Comm. Alg. 22 (1994), no. 12, 4737-4749.

[3] P. Crawley and B. Jonsson, Refinements for infinite direct decomposition of algebraic systems, Pacific. Math. 14 (1964), 797-855.

[4] K. R. Goodearl, von Neuman Regular Rings, Pitman, 1979.

[5] C. Y. Hong, N. Y. Kim, and Y. Lee, Exchange rings and their extensions, J. Pure and Appl. Alg. 179 (2003), 117-126.

[6] T. Y. Lam, A First Course in Noncommutative Rings, Springer-Verlag, 1991.

[7] W. K. Nicholson, I-rings, Trans. Amer. Math. Soc. 207 (1975), 361-373.

[8] __ Lifting idempotents and exchange rings, Trans. Amer. Math. Soc. 299 (1977), $269-278$.

[9] J. Stock, On rings whose projective modules have exchange property, J. Alg. 103 (1986), $437-453$

[10] R. B. Jr. Warfield, Exchange rings and deposition modules, Math. Ann. 199 (1992), 31-36.

[11] H. P. Yu, On the structure of exchange rings, Comm. Alg. 25 (1997), no. 2, 661-670.

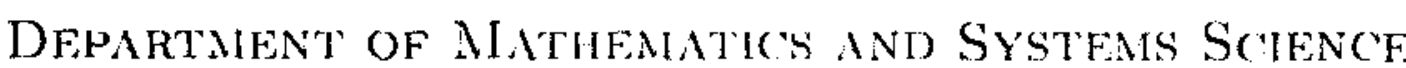

National University' of Defense 'Peghnology Changsha

HLNAN 410073, CHINA

E-mail address: ydlbj@yahoo.com.cn 